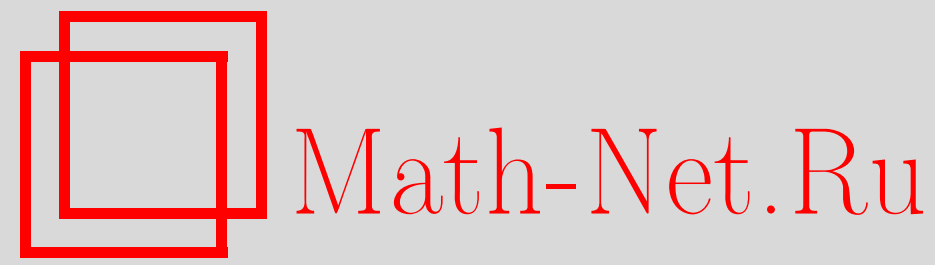

Д. О. Крамков, О замыкании семейства мартингальных мер и опциональном разложении супермартингалов, Теория вероятн. и ее примен., 1996, том 41, выпуск 4, 892-896

DOI: https://doi.org/10.4213/tvp3242

Использование Общероссийского математического портала Math-Net.Ru подразумевает, что вы прочитали и согласны с пользовательским соглашением http://www . mathnet.ru/rus/agreement

Параметры загрузки:

IP : 54.162 .85 .209

26 апреля 2023 г., 13:36:52

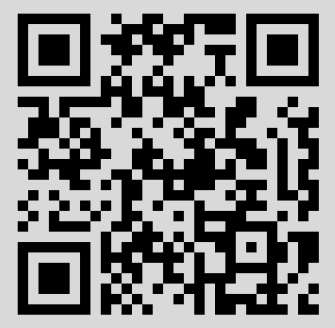


13. Фрадков $A$. Л., Якубович $B . A . S$-процедура и соотношение двойственности в невыпуклых задачах математического программирования. - Вестн. Ленинградского ун-та, 1973, т. 27, № 1, с. 71-76.

14. Якубович $B$. A. Об одном методе решения задач глобальной минимизации. Вестн. С.-Петербургского ун-та, сер. I, 1992, № 2, с. 58-68.

15. Yakubovich V.A. Nonconvex optimization problem: the infinite-horizon linear quadratic control problem with quadratic constraints. - Systems Control Lett., 1992, v. 16, № 6, p. $13-22$.

16. Докучаев Н. Г., Якубович $B . A$. Стохастическая линейно-квадратическая задача оптимального управления для стационарных систем с квадратичными ограничениями. - Техн. кибернет., 1992, № 6, с. 135-145.

17. Матвеев A. C., Якубович B. A. Невыпуклые задачи глобальной оптимизации. Алгебра и анализ, 1992, т. 4, № 6, с. 189-219.

18. Докучаев $H . \Gamma . S$-процедура для нелинейных обыкновенных дифференциальных уравнений с стохастически распределенным начальным значением. - Вестн. С.-Петербургского ун-та, сер. 1, 1994, № 3, с. 117-120.

19. Borkar S. V., Ghosh M. K. Controlled diffusions with constraints. - J. Math. Anal. Appl., 1990, v. 152, № 1, p. 88-108.

20. Фрид $E$. $Б$. Об оптимајьных стратегиях в задачах управления с ограничением. Теория вероятн. и ее примен., 1972, т. XVII, в. 1, с. 194-199.

21. Кудрявчев Д. И. Управление марковскими процессами в задаче с ограничениями. - Укр. матем. журн., 1989, т. 41, № 9, с. 1226-1230.

22. Пиумовский $A$. Б. Управление случайными последовательностями в задачах с ограничениями. - Теория вероятн. и ее примен., 1993, т. 38, в. 4, с. 891-903.

Поступила в редакцию 12.VII.1994

(C) $1996 \mathrm{r}$.

КРАМКОВ Д. О.*

\title{
О ЗАМЫКАНИИ СЕМЕЙСТВА МАРТИНГАЛЬНЫХ МЕР И ОПЦИОНАЛЬНОМ РАЗЛОЖЕНИИ СУПЕРМАРТИНГАЛОВ ${ }^{1)}$
}

\begin{abstract}
Описана структура замыкания семейства мартингальных мер в топологии пространства $L_{1}$. Полученный результат использован для нового доказательства теоремы об опциональном разложении супермартингалов.
\end{abstract}

Ключевые слова и Фразы: мартингальная мера, опциональное разложение супермартингалов, семимартингал, сходимость по Фату.

1. Введение и формулировка основного результата. Пусть $X$-локальный мартингал со значениями в $\mathbf{R}^{d}$, заданный на стохастическом базисе $(\Omega, \mathcal{F}$,

*Математический ин-т им. В. А. Стеклова РАН, ул. Вавилова 42, 117966 Москва, ГСП-1, Россия.

1) Работа выполнена при поддержке Российского фонда фундаментальных исследований, грант 96-01-01929. 
$\left.\left(\mathcal{F}_{t}\right)_{t \geqslant 0}, \mathbf{P}\right)$. Вероятностную меру $\mathbf{Q} \sim \mathbf{P}$ назовем эквивалентяой1) локалькой мартингальной мерой (процесса $X$ ), если $X$ является локальным мартингалом относительно $\mathbf{Q}$. Семейство всех эквивалентных мартингальных мер обозначим $\mathcal{M}=$ $\mathcal{M}(X, \mathbf{P})$. Определим также семейство мер $\mathcal{M}^{0}=\mathcal{M}^{0}(X, \mathbf{P}):$

$$
\mathcal{M}^{0}=\mathcal{M}^{0}(X, \mathbf{P})=\left\{\mathbf{Q} \in \mathcal{M}: \max \left(\left\|\frac{d \mathbf{Q}}{d \mathbf{P}}\right\|_{\infty},\left\|\frac{d \mathbf{P}}{d \mathbf{Q}}\right\|_{\infty}\right)<+\infty\right\} .
$$

Иными словами, $\mathcal{M}^{0}$ есть подмножество $\mathcal{M}$, состоящее из таких мер $\mathbf{Q} \in \mathcal{M}$, что нормы пространств $L^{1}(\mathbf{Q})$ и $L^{1}(\mathbf{P})$ эквивалентны. Для произвольного семейства семимартингалов $\mathcal{S}$ обозначим $\mathcal{P}(\mathcal{S})$ (соответственно, $\widehat{\mathcal{P}}(\mathcal{S})$ ) семейство вероятностных мер $\mathbf{Q} \sim \mathbf{P}$ (соответственно, $\mathbf{Q} \ll \mathbf{P}$ ) таких, что все $S \in \mathcal{S}$ являются локальными супермартингалами относительно $\mathbf{Q}$.

Введем теперь в рассмотрение семейство $\mathcal{S}^{*}$ локально ограниченных снизу семимартингалов $\mathcal{S}$, являющихся стохастическими интегралами по $X$ :

$$
\mathcal{S}^{*}=\left\{S=\int H d X: \int H d X: \text { локально ограничен снизу }\right\} .
$$

Отметим, что всякий $S \in \mathcal{S}^{*}$ является локальным мартингалом относительно $\mathbf{Q} \in \mathcal{M}$, см. [6] и [1]. Тем самым

$$
\mathcal{M}^{0} \subset \mathcal{M} \subset \mathcal{P}\left(\mathcal{S}^{*}\right) \subset \widehat{\mathcal{P}}\left(S^{*}\right)
$$

Отметим также, что если процесс $X$ локально ограничен, то $\mathcal{P}\left(\mathcal{S}^{*}\right)$ (соответственно, $\widehat{\mathcal{P}}\left(\mathcal{S}^{*}\right)$ ) совпадает с семейством всех эквивалентных (соответственно, абсолютно непрерывных) локальных мартингальных мер.

Сформулируем основной результат работы.

Теорема 1.1. Семейство мер $\widehat{\mathcal{P}}\left(\mathcal{S}^{*}\right)$ яөлнется замыканием семейств $\mathcal{M}^{0}$ и $\mathcal{M}$ в топологии пространства $L^{1}(\mathbf{P})$.

3 а м е ч а н и е 1.1. Поскольку семейства $\mathcal{M}^{0}$ и $\mathcal{M}$ являются выпуклыми, их замыкания в сильной и слабой топологии совпадают.

Теорема 1.1 уточняет теорему 2.1 в [6]. В п. 3 с ее помощью будет получено новое доказательство теоремы об опциональном разложении супермартингалов без услови $x$ локальной ограниченности на процесс $X$. Отметим, что семейство мартингальных мер играет исключительно важную роль в финансовой математике, см., например, [2].

2. Доказательство теоремы 1.1. Ключевую роль при доказательстве теоремы 1.1 играет один факт относительно сходимости по Фату для последовательности случайных процессов. Прежде всего, дадим следующее определение.

O п р е д е л е н и е 2.1. Пусть $\mathcal{T}$ - плотное подмножество $\mathbf{R}_{+}$. Последовательность случайных процессов $\left(X^{n}\right)_{n} \geqslant 1$ сходится по Фату к процессу $X$ ма множестве $\mathcal{T}$, если она равномерно ограничена снизу и

$$
X_{t}=\limsup _{s \downarrow t, s \in T} \limsup _{n \rightarrow \infty} X_{s}^{n}=\liminf _{s \downarrow t, s \in T} \liminf _{n \rightarrow \infty} X_{s}^{n}
$$

почти наверное, при всех $t \geqslant 0$.

Следуя [5], семейство семимартингалов $\mathcal{S}$ назовем предсказуемо выпуклым, если наряду с любыми $S^{1}$ и $S^{2}$ оно содержит и стохастический интеграл $\int\left(\alpha d S^{1}+(1-\right.$ $\alpha) d S^{2}$ ) для произвольного предсказуемого $0 \leqslant \alpha \leqslant 1$.

1) Если $\mathbf{Q}$ и $\mathbf{R}$ - две вероятностные меры, заданные на измеримом пространстве $(\Omega, \mathcal{F})$, то запись $\mathbf{Q} \ll \mathbf{R}$ обозначает, что $\mathbf{Q}$ абсолютно непрерывна относительно $\mathbf{R}:(\forall A \in \mathcal{F}) \mathbf{R}(A)=0 \Longrightarrow \mathbf{Q}(A)=0$. Меры $\mathbf{Q}$ и $\mathbf{R}$ называются эквивалентными (обозначение $\mathbf{Q} \sim \mathbf{R})$, если $(\forall A \in \mathcal{F}) \mathbf{R}(A)=0 \Longleftrightarrow \mathbf{Q}(A)=\mathbf{0}$. 
Пусть теперь $\mathcal{S}$ - предсказуемо выпуклое семейство локально ограниченных снизу семимартингалов, которое является конусом и содержит все невозрастающие локально ограниченные процессы (в частности, процесс $S \equiv 0$ ). Следуюшее утверждение является частным случаем предложения 5.2 в [5].

Предложение 2.1. Предположим, ито $\mathcal{P}(\mathcal{S}) \neq 0$ и множество $\mathcal{S}$ замкнуто относительно сходимости Фату для некоторого всюду плотного множества $T$. Тогда локально ограниченный снизу прочесс $V$ прияадлежит $\mathcal{S}$, если и только если для всех $\mathbf{Q} \in \mathcal{P}(\mathcal{S})$ процесс $V$ является локальньм супермартингалом относительно меры $\mathbf{Q}$.

Перейдем теперь к доказательству теоремы 1.1.

Д ок аз а те ль с т в о те о р е м ы 1.1. Нетрудно проверить, что множество $\widehat{\mathcal{P}}\left(\mathcal{S}^{*}\right)$ замкнуто в $L^{1}$. Тем самым достаточно показать, что замыкание $\mathcal{M}^{0}$ в $L^{1}$ содержит $\widehat{\mathcal{P}}\left(\mathcal{S}^{*}\right)$. Поскольку множества $\mathcal{M}^{0}$ и $\widehat{\mathcal{P}}\left(\mathcal{S}^{*}\right)$ выпуклы и $\mathcal{M}^{0} \subset \widehat{\mathcal{P}}\left(\mathcal{S}^{*}\right)$, в силу теоремы Хана-Банаха это имеет место, если

$$
\sup _{\mathbf{Q} \in \mathcal{M}^{0}} \mathbf{E}_{\mathbf{Q}^{h}}=\sup _{\mathbf{Q} \in \widehat{\mathcal{P}}\left(\mathcal{S}^{*}\right)} \mathbf{E}_{\mathbf{Q}^{h}}
$$

для любой ограниченной случайной величины $h$. Для доказательства (1) определим процесс

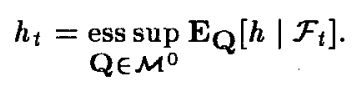

Процесс $\left(h_{t}\right)_{t \geqslant 0}$ является супермартингалом относительно всех $\mathbf{Q} \in \mathcal{M}^{0}$. Доказательство данного факта дословно повторяет доказательство предложения 4.3 в [7] и здесь опускается. Тогда (1) имеет место, если $h$ - супермартингал относительно Bcex $\mathbf{Q} \in \mathcal{P}\left(\mathcal{S}^{*}\right)$.

Введем в рассмотрение семейство $\mathcal{R}$ семимартингалов $R$ следующего вида: $R=$ $a(Z-1)-C$, где $Z$ - процесс плотности элемента $\mathcal{M}^{0}, a \in \mathbf{R}$ и $C$ - локально ограниченный возрастающий процесс. Обозначим $\overline{\mathcal{R}}$ наименьшее множество семимартингалов, которое предсказуемо выпукло, замкнуто относительно сходимости Фату на множестве рациональных чисел и содержит $\mathcal{R}$. В силу леммы Фату процесс $R h$ является локальным супермартингалом для любых $R \in \overline{\mathcal{R}}$. Таким образом, нам достаточно доказать справедливость вложения

$$
\mathcal{P}\left(\mathcal{S}^{*}\right) \subset \overline{\mathcal{R}}
$$

Для доказательства (2) воспользуемся предложением 2.1. Зафиксируем меру $\mathbf{Q} \in \mathcal{P}(\overline{\mathcal{R}})$ и обозначим $Z^{Q}$ ее процесс плотности относительно меры $\mathbf{P}$. Поскольку $\overline{\mathcal{R}}$ содержит линейное пространство, порождаемое процессами плотности элементов $\mathcal{M}^{0}, \mathbf{Q}$ является мартингальной мерой для процессов плотности элементов $\mathcal{M}^{0}$, или, что равносильно, $Z^{Q}$ - мартингал относительно всех мер из $\mathcal{M}^{0}$. Но тогда по теореме Jacka [6] $Z^{Q}$ есть стохастический интеграл относительно $X: Z^{Q}=1+\int H d X$, и, следовательно, является супермартингалом относительно всех мер из $\mathcal{P}\left(\mathcal{S}^{*}\right)$. В силу произвольности $\mathbf{Q} \in \mathcal{P}(\overline{\mathcal{R}})$, это влечет за собой выполнение условий предложения 2.1 для любого элемента множества $\mathcal{P}\left(\mathcal{S}^{*}\right)$ и, следовательно, (2). Теорема доказана.

3. Опциональное разложение супермартингалов. Пелью данного раздела является доказательство следующего результата.

Теорема 3.1 (опциональное разложение супермартингала). Пусть $V-$ неотрицательный супермартинаал относительно всех $\mathbf{Q} \in \mathcal{M}^{0}(X ; \mathbf{P})$. Тогда майдутся возрастаючий согласованяый прочесс $C$ и предсказуемый $X$-интегрируемый nроцесс $H$ maxue, чmo

$$
V=V_{0}+\int H d X-C
$$


Стохастический интеграл $\int H d X$ в (3) является локальным мартингалом относительно всех $\mathbf{Q} \in \mathcal{M}^{0}$. Тем самым (3) есть представление супермартингала $V$ в виде разности между локальным мартингалом и возрастающим процессом, выполненное одновременно при всех $\mathbf{Q} \in \mathcal{M}^{0}$. В частном случае, когда семейство $\mathcal{M}^{0}$ состоит из единственного элемента, оно сводится к классическому результату Дуба-Мейера. Отметим, однако, что, в отличие от последнего, разложение (3), вообще говоря, не существует при дополнительном требовании о том, чтобы процесс $C$ являлся предсказуемым. По этой причине его называют опциональным разложением супермартингала $V$.

Представления типа (3) играют важную роль в задачах финансовой математики, см. [3], [7], [8], [5]. Впервые они были получены в [3] для процесса $X$ диффузионного типа и для конкретного вида процесса $V$. В том случае, когда $X$ - произвольный локально ограниченный семимартингал, теорема 3.1 была доказана в [7]. Наконец, в общем случае она была доказана в [4]. Укажем также на работу [5], где рассматривалась возможность представления семимартингала $V$ в виде (3) при наличии ограничений на интегрируемый процесс $H$. Отметим, что в указанных работах требовалось, чтобы $V$ являлся супермартингалом относительно всех мер из семейства $\mathcal{M}(X, \mathbf{P})$, хотя и не предполагалось, что $X$ является локальным мартингалом относительно «базовой» меры $\mathbf{P}$.

Доказательство в [4] использует теорему Гирсанова и теорему об измеримом выборе. Ниже мы приведем другое доказательство теоремы об опциональном разложении, используя теорему 1.1 и некоторые результаты работы [5] относительно Фату сходимости.

Д ок а з а т ел с с т в о. Введем в рассмотрение семейство $\mathcal{S}$ локально ограниченных снизу семимартингалов $S$ вида

$$
S=\int H d X-C,
$$

где $C$ - возрастающий процесс. В силу предложения 2.1 разложение (3) имеет место, если

1) $V$ - супермартингал относительно всех $\mathbf{Q} \in \mathcal{P}(\mathcal{S})$,

2) семейство $\mathcal{S}$ замкнуто относительно сходимости Фату.

В силу теоремы 1.1 и леммы Фату процесс $S$ является супермартингалом относительно всех $\mathbf{Q} \in \mathcal{P}\left(\mathcal{S}^{*}\right)$. Теперь 1) следует из того факта, что $\mathcal{P}\left(\mathcal{S}^{*}\right)=\mathcal{P}(\mathcal{S})$. Пункт 2) доказан в [5], см. предложение 6.1 и замечание после предположения 3.1.

Автор благодарен Ю. М. Кабанову за многочисленные обсуждения и замечания.

\section{СПИСОК ЛИТЕРАТУРЫ}

1. Ansel J. P., Stricker C. Couverture des actifs contingents. - Ann. Inst. H. Poincaré, 1994 , v. 30, № 2, p. 303-315.

2. Delbaen $F$., Schachermayer $W$. A general version of the fundamental theorem of asset pricing. - Math. Ann., 1994, v. 300, p. 463-520.

3. El Karoui $N$., Quenez $M$ : C. Dynamic programming and pricing of contingent claims in an incomplete market. - SIAM J. Control Optim., 1995, v. 33, № 1, p. 29-66.

4. Föllmer H., Kabanov Yu. M. Optional decompositions and Lagrange multipliers. Preprint, 1996.

5. Föllmer H., Kramkov D. O. Optional decompositions under constraints. - Preprint, 1996.

6. Jacka S. D. A martingale representation result and an application to incomplete financial markets. - Math. Finance, 1992, v. 2, № 4, p. 239-250. 
7. Kramkov D. O. Optional decomposition of supermartingales and hedging contingent claims in incomplete security markets. - Probab. Theory Relat. Fields, 1996, v. 105, p. $459-479$.

8. Kabanov Yu. M., Kramkov D. O. Asymptotic arbitrage in large financial markets. Finance and Stochastics (to appear).

Поступила в редакцию 18. VII. 1996

(C) $1996 \mathrm{r}$.

СТЕПАНОВ А. В.*

\section{ЭКСТРЕМАЛЬНЫЕ ПОРЯДКОВЫЕ СТАТИСТИКИ ПРРИ ИЗМЕНЕНИИ ОТНОШЕНИЯ ПОРЯДКА}

Пусть $X_{1}, X_{2}, \ldots$ - независимые случайные величины с обшей непрерывной функцией распределения $F(x)$. Фиксируем произвольную точку а на носителе вероятностной меры. Будем рассматривать последовательности случайных величин, состоящие из тех величин $X_{i}$, которые в той или иной степени стремятся к точке $a$. Кроме того, будем изучать индексы зтих случайных величин. По подходам и методам решений данная проблематика напоминает теорию рекордов.

Ключевые слова и фразы: рекордные моменты и величины, экстремальные порядковые статистики, последовательности случайных величин, приближающиеся к заданной точке.

1. Введение. Пусть здесь и везде далее $X_{1}, X_{2}, \ldots$ - независимые случайные величины (с.в.) с общей непрерывной функцией распределения $F(x)$. Пусть интервал $(\gamma, \beta)$ - носитель вероятностной меры, где $\gamma$ может быть равным $-\infty$, а $\beta$ может принимать значение $+\infty$. Рассмотрим с.в. $X_{1}, X_{2}, \ldots, X_{n}$ в неубывающем порядке: $\gamma<X_{1, n}<X_{2, n}<\cdots<X_{n, n}<\beta$. Величины $X_{r, n}(r \leqslant n)$ называют порядковыми статистиками. «Согнем» интервал $(\gamma, \beta)$ в окружность, соединив точки $\gamma$ и $\beta$. При такой постановке вопроса большей является та случайная величина, которая находится далее при обходе окружности вдоль часовой стрелки с началом обхода в точке $\gamma$ и концом обхода в точке $\beta$. Выберем теперь на окружности некоторую точку $a$ и зафиксируем ее. Начало обхода сдвинем в эту точку, а направление обхода сохраним. В этом случае вместо $\gamma$ мы имеем $a+0$, а вместо $\beta$ необходимо брать $a-0$. Отношение порядка среди с.в. $X_{i}$ изменится. Так, например, если с.в. располагались следующим образом:

$$
\gamma<X_{1, n}<X_{2, n}<\cdots<X_{n-2, n}<a<X_{n-1, n}<X_{n, n}<\beta,
$$

и если $\widetilde{X}_{r, n}$ - новые порядковые статистики, т.е.

$$
X_{n-1, n}=\widetilde{X}_{1, n}, \quad X_{n, n}=\widetilde{X}_{2, n}, \quad X_{1, n}=\widetilde{X}_{3, n}, \quad X_{n-2, n}=\widetilde{X}_{n, n},
$$

то $a+0 \prec \tilde{X}_{1, n} \prec \tilde{X}_{2, n} \prec \beta=\gamma \prec \tilde{X}_{3, n} \prec \cdots \prec \tilde{X}_{n, n} \prec a-0$. Здесь знаком $\prec$ обозначаем новое отношение порядка (см. также рис. 1).

Рассмотрим построенные определенным образом и связанные с экстремальными порядковыми статистиками последовательности с.в.: последовательности величин односторонних последовательных приближений («о.п.п.») $V_{(n)}^{a}$, моментов «о.п.п.»

*Калининградский государственный технический университет, Советский проспект, 1, 236000 Калининград, Россия. 